\title{
Incidencia de las políticas empresariales medioambientales en la gestión de residuos sólidos en el sector construcción, Lima 2018
}

Alberto Mendiola LÁzaro de Ortecho

Recibido: 04/06/2020 Aceptado: 15/10/2020 Publicado: 31/12/2020

\begin{abstract}
RESUMEN
Un problema de las grandes ciudades y por lo tanto de Lima, es la afectación de su medio ambiente debido a los residuos sólidos generados por las actividades constructivas. El presente estudio busca demostrar que se puede hacer una buena gestión integral de los residuos sólidos a través de la aplicación de políticas empresariales medioambientales efectivas con reglas y normas y no simple declaraciones de buena intensión. El instrumento de medición es un cuestionario plasmado en una encuesta a 86 profesionales del sector construcción en la ciudad de Lima y de cuyos resultados se han elaborado dos tipos de análisis; uno estadístico que demostró la correlación positiva considerable (incidencia) entre las dos variables y otro con aportes de los entrevistados para una buena gestión integral de los residuos sólidos en las obras.
\end{abstract}

Palabras clave: Políticas empresariales medioambientales; gestión integral de residuos sólidos.

INCIDENCE OF ENVIRONMENTAL BUSINESS POLICIES IN THE MANAGEMENT OF SOLID WASTE IN THE CONSTRUCTION SECTOR, LIMA 2018

\section{ABSTRACT}

A problem of the big cities and, therefore, of Lima, is the damage to the environment because the solid residues generated by construction activities. The present study seeks to demonstrate that a good and comprehensive solid waste management can be done through the application of effective environmental business policies, with rules and regulations, and not simple statements of good intention. The means of measurement is a questionnaire captured in a survey of 86 professionals from the construction sector in Lima, and which results have produced two types of analysis: one statistical that demonstrated the significant positive correlation (incidence) between the two variables, and the other one with contributions from the interviewees for good, comprehensive solid waste management in the jobsite.

Keywords: Environmental business policies; Integral management of solid waste.

\section{INTRODUCCIÓN}

La contaminación del medio ambiente en las ciudades afecta la salud de las personas a través del deterioro de los recursos naturales como el agua, aire y suelos. Este es un tema del que muchos autores han escrito planteando diferentes orígenes al problema, en este artículo discutiremos uno de ellos, el referido a los residuos sólidos en los proyectos de construcción en la ciudad de Lima.

El presente estudio revisa el problema de los residuos sólidos que se producen en la ciudad de Lima a consecuencia de los proyectos de construcción y busca demostrar que las políticas empresariales medioambientales de las empresas constructoras, implementadas correctamente, tienen una inciden positiva en la gestión de los residuos sólidos de la ciudad. Siendo este el objetivo principal del estudio y por lo cual se planteó la hipótesis "Las políticas empresariales medioambientales inciden en la gestión integral de residuos sólidos en las obras del sector construcción en Lima Metropolitana".

Al revisar la literatura existente de estas dos variables se encuentra que es abundante debido a que son temas de actualidad, lo que si se observó es que no hay un análisis de estas dos variables en conjunto. Koontz \& O'Donnell, (1972) define "Las políticas son planteamientos generales o maneras de comprender, que guían o canalizan el pensamiento y la acción en la toma de decisiones de todos los miembros de la organización", tomando este concepto, en las empresas está muy desarrollado el uso de políticas empresariales medioambientales, como un modo de orientar el comportamiento de sus colaboradores con respecto al medio que nos rodea. Con respecto a los residuos sólidos, hay mucha literatura orientada principalmente a los orígenes domésticos que al que genera el sector construcción, es por ello la importancia de asociar estas dos variables y ver si las políticas empresariales inciden en la gestión de los residuos sólidos en los proyectos constructivos.

1 Ingeniero Industrial (CIP 28158) de la Universidad de Lima, profesor a tiempo parcial de posgrado de la Universidad Peruana de Ciencias Aplicadas, especialista en Supply Chain Management en proyectos de construcción, Lima, Perú. ORCID: https://orcid.org/0000-0002-3271-4097 E-mail: a mendiola1@hotmail.com 
Profundizando en el tema y analizando su origen se observa que el desarrollo urbanístico desordenado y la falta de control del estado en la gestión de los residuos sólidos en el sector construcción, principalmente en las etapas de eliminación, afecta el medio ambiente de la ciudad. En Lima, la mala gestión de los residuos como en toda ciudad grande, causa problemas ambientales serios como la contaminación de sus playas en la Costa Verde, el río Rímac, al lado de las pistas, y otras zonas urbanas, tomados como botaderos de desmonte y otros materiales.

Cuantificando el problema, según el INEI (2018), hasta ese año la población de la ciudad de Lima era de 9.32 millones de habitantes con una superficie de 2672 km2 (no incluye la provincia constitucional del Callao), por otro lado, AMBIDES (2012) en su Informe Anual de Residuos Sólidos Municipales y no Municipales en el Perú, Gestión 2012 del Ministerio del Ambiente señala:

El Sector Vivienda y Saneamiento indica como sus principales residuos una clasificación indefinida (tierra y polvo de construcción, residuos de madera y afines), con un porcentaje de $96.14 \%$; el segundo tipo de residuo de mayor importancia son los residuos de construcción, con un $3.58 \%$ (p. 17-18).

El Ministerio de Vivienda no tiene una definición o clasificación para el $96.14 \%$ de los residuos que se generan en su sector, de ahí parte el problema, ya que sin una clasificación correcta de los residuos como se podría identificar los materiales que pueden ser reprocesados y legislar sobre el tema. En este caso la clasificación queda cargo de las empresas constructoras los cuales sin mayor esfuerzo lo tiran a la basura, sin darse cuentas que están perdiendo dinero. Este problema es conocido de muchos años atrás, Durand y Metzger (2009) en su informe "Gestión de residuos y transferencia de vulnerabilidad en Lima/Callao" mencionaban lo siguiente:

Finalmente, los residuos de la construcción son responsabilidad de cada empresario, de cada constructor. Sin vertedero oficial de escombros en la aglomeración (se está preparando una ley sobre el tema), estos terminan en los botaderos ubicados en los lugares abandonados de la ciudad: a lo largo de los ríos, en las playas, en la berma central de las avenidas, en las antiguas canteras o sobre los pendientes de los cerros de la ciudad (p.11).
El Ministerio del Ambiente ha reglamentado la disposición de los residuos sólidos del sector construcción a través del D.L. 1278, Ley de Gestión Integral de Residuos Sólidos y su reglamento el DS 014-2017-MINAN del Ministerio del Ambiente (2017), el cual señala en su artículo 42, Disposición final de residuos sólidos municipales, lo siguiente: "La disposición final de los residuos sólidos peligrosos, no peligrosos y residuos provenientes de actividades de la construcción y demolición de gestión municipal deben realizarse en celdas diferenciadas", y lo complementa indicando en la parte de "Aspectos generales":

Los residuos sólidos no peligrosos provenientes de las actividades de la construcción y demolición deben disponerse en escombreras o rellenos sanitarios que cuenten con celdas habilitadas para tal fin. El Ministerio de Vivienda, Construcción y Saneamiento regula las condiciones y características de las escombreras (artículo 69).

Entiéndase por celdas a la "infraestructura ubicada dentro de un relleno sanitario donde se esparcen y compactan finalmente los residuos depositados" (D.L. 1278, Definiciones, 2017).

Por otro lado, el Observatorio Ciudadano Lima cómo Vamos (2015, p.15), en su informe Evaluando la Gestión de Lima - Quinto Informe de Resultados sobre Calidad de Vida, señala que en el 2013 Lima y Callao han producido alrededor de 7452.67 toneladas de residuos sólidos municipales al día. El volumen de residuos sólidos de ese mismo año (ver tabla 1) en construcción fue de 1581.06 miles de $\mathrm{m} 3$ de un total de 1705.4 miles de $\mathrm{m} 3$, es decir, el $92.7 \%$ del total, siendo la mayor parte residuos minerales (1485.9 miles de $\mathrm{m} 3$ ) los cuales pueden ser reprocesados.

Como se muestra en el cuadro, la industria de la construcción produce un volumen importante de residuos sólidos en la ciudad de Lima, la cual contamina el medio ambiente, de ahí la importancia de evitar que los proyectos generen residuos sólidos innecesarios, y los que se produzcan sean gestionados correctamente. De ahí la importancia del estudio al plantear como parte de la solución a este problema que las empresas constructoras diseñen Políticas Empresariales Medioambientales (PEM), debidamente expuestos y aplicados en forma responsable y eficientemente, de tal manera que afecten positivamente la Gestión Integral de los Residuos Sólidos (GIRS) en los diferentes proyectos de construcción. 
Tabla 1. Volumen y composición de los Residuos de Construcción y Demoliciones (RCD)

\begin{tabular}{|c|c|c|c|c|c|c|c|c|}
\hline & Total & \multicolumn{2}{|c|}{ Residuos de la construcción y demoliciones (RCD) } & \multicolumn{3}{c|}{ Otros residuos (no RCD) } \\
\cline { 3 - 10 } & & Minerales & $\begin{array}{c}\text { Otros no } \\
\text { peligrosos }\end{array}$ & $\begin{array}{c}\text { Madera } \\
\text { tratada }\end{array}$ & Otros peligrosos & Domiciliarios & No domiciliarios & $\begin{array}{c}\text { Residuos } \\
\text { peligrosos }\end{array}$ \\
\hline $\begin{array}{c}\text { Volumen m3 } \\
\text { (miles) }\end{array}$ & 1705.4 & 1485.9 & 59.3 & 13.46 & 22.4 & 110.0 & 13.8 & 0.5 \\
\hline Porcentaje & $100.0 \%$ & $87.1 \%$ & $3.5 \%$ & $0.8 \%$ & $1.3 \%$ & $6.5 \%$ & $0.8 \%$ & $0.0 \%$ \\
\hline
\end{tabular}

Fuente: Plan de incentivos a la mejora de la Gestión y Modernización Municipal, Ministerio de Vivienda, Construcción y Saneamiento Arq. Marissa Andrade G. 2014

Para la variable políticas empresariales medioambientales es importante analizarla desde las dimensiones de su sostenibilidad y sustentabilidad ambiental en el tiempo, la sensibilidad y educación ambiental que deben promover las empresas, así como, la responsabilidad social empresarial.

Con respecto a la sostenibilidad y sustentabilidad en el tiempo, Larrouyet (2015) indica:

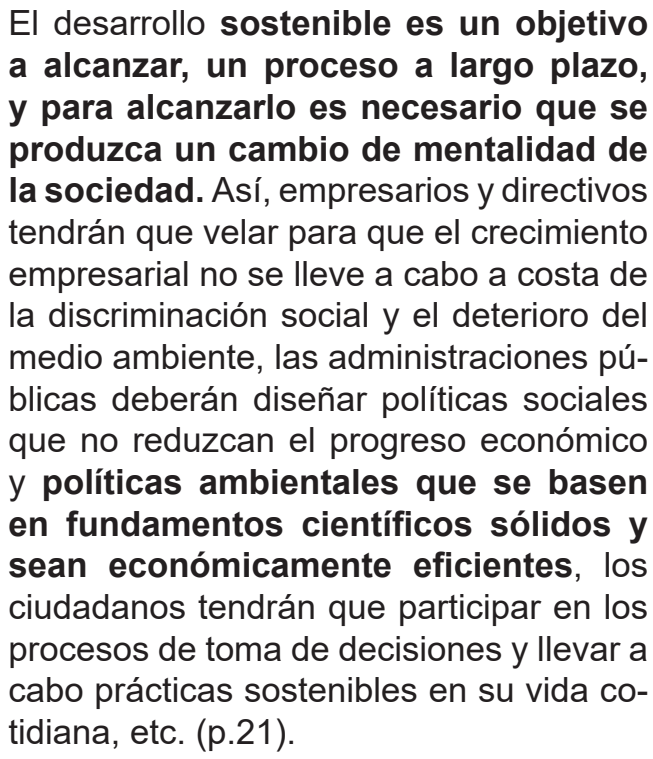

Por otro lado, Dourojeanni (1999) sostiene que "solo si el desarrollo sustentable se mantiene en el tiempo se alcanza el denominado desarrollo sostenible" (p.1), es decir, si las PEM tienen incidencia positiva en la GIRS, estas deben ser permanente en la organización y a la vez flexibles con los cambios que se den en el futuro (conceptuales, tecnológicos y otros).

Otra característica de las PEM es de sensibilizar a los colaboradores con el medioambiente que los rodea, Rivas (2011) señala que a través de la implementación de sistemas de calidad como el ISO con la norma ISO 1400 "se debe velar por la regulación de esta y considerar las demandas de los consumidores y la sensibilidad de la comunidad frente a temas medioambientales como la contaminación, el reciclaje, el agua y los problemas relacionados" (p.158). También es importante estar permanentemente educándolos en estos temas e incentivándolos en la búsqueda de niveles de calidad en el desarrollo de sus actividades. Aquí es importante la participación de la alta gerencia la cual debe estar comprometida y asignar los recursos necesarios para el éxito del proyecto, como indica el autor anteriormente mencionado:

Los sistemas de gestión efectivos son aquellos que no solo demuestran su compromiso con el medio ambiente, sino que, además, son eficientes en su desarrollo y para ello, es necesario que haya un compromiso de la dirección de la Institución: este compromiso es entendido como la dedicación de recursos tales como tiempo y dinero a la implementación del nuevo sistema. Además, debe estar planteado un proceso de mejora continuada en cuanto a medidas preventivas y correctivas, formación de la comunidad y auditorías internas o seguimiento (p.158).

Otro punto está en el análisis del grado de compromiso con la Responsabilidad Social Ambiental de las empresas constructoras y si existe una estructura que lo soporta, esto permitiría que sus colaboradores sean proactivos e innovadores en el tema de la GIRS, Pulgar (2010) señala lo siguiente:

Una empresa o entidad socialmente responsable cuenta con una estructura organizativa que facilita la participación, la innovación, los aportes; se articula con el personal de manera de generar relaciones equitativas, informadas; tiene como meta central la protección ambiental y la prevención de impactos potenciales de su actividad; es proactiva y está en constante 
búsqueda de nuevas soluciones frente a nuevos retos; y es administrativamente flexible y adaptable a responsabilidades que resultan transversales (p.222).

Ser una empresa socialmente responsable con el medio ambiente, no necesariamente debe representar un costo para la organización, en el caso de las empresas de construcción muchos de los materiales residuales pueden ser reciclados, recuperando perdidas e incluso produciendo ganancias, como lo menciona Rodriguez (2018):

The notion of Environmental Corporate Social Responsibility has emerged because of the progressive increase of environmental concerns at international, European and national levels such as the challenge of climate change. Green initiatives are not seen as a "cost", but as a new opportunity to make more profits and transform a polluting activity into a greener one (p.2).
La otra variable es la GIRS, que son todas las actividades que contemplan el manejo de los residuos sólidos, desde su generación hasta su eliminación, pasando por las etapas de ubicación, clasificación, recuperación y transporte. Estas mismas etapas se dan en sector construcción con algunas pequeñas variantes debido a la variedad de los proyectos, así como la ubicación de estos. No es lo mismo hacer un edificio en Lima, que es la capital, que hacerlo en provincia, los procesos y los tipos de materiales pueden ser diferentes, por lo tanto, producen residuos diferentes y su tratamiento para su eliminación varia.

Con el fin de identificar mejor los residuos sólidos en un proyecto de construcción se ha elaborado la figura 1 que muestra un flujograma de las diferentes etapas por las que debe pasar los residuos. Esta va desde su creación en los proyectos, pasando por la clasificación para determinar que residuos pueden ser procesables o recuperables, como el fierro de construcción, los agregados etc. y cuáles deben ser enviados a los rellenos sanitarios oficiales. También

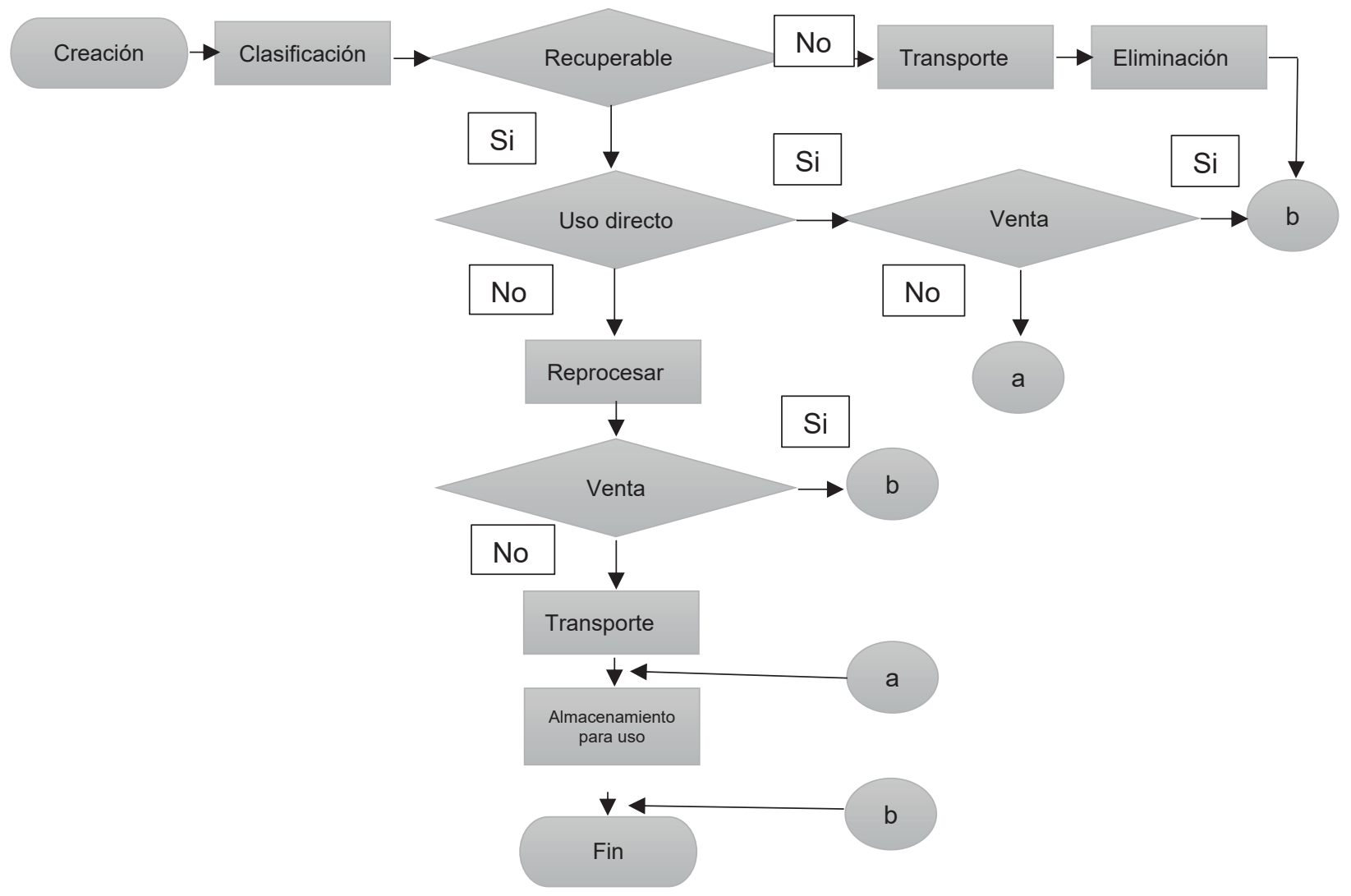

Figura 1. Flujograma de la gestión de residuos sólidos en el sector construcción Fuente: Elaboración propia 
hay una serie de materiales que son sobrantes de la obra (se generan por un mal diseño, malas especificaciones técnicas o mal metrado) que pueden ser reusados en otros proyectos o vendidos con el fin de recuperar algo de la inversión.

La ONU en su Programa de las Naciones Unidas para el Medio Ambiente (PNUMA), plantea los principios que debe tener las políticas medioambientales, los cuales deben crearse basándose en los "compromisos ambientales" que deben asumir las empresas siendo uno de ellos la de minimizar en lo posible la emisión de residuos sólidos. Es por ello, que para este estudio es importante determinar que las PEM de las empresas constructoras cumplen con este propósito, a través de una buena GIRS, es decir, las PEM deben incidir positivamente en las GIRS. Esta incidencia positiva se da con la reducción de los residuos o su eliminación en lugares ya establecidos por el estado, evitando la contaminación y se proteja el medio ambiente de la ciudad de Lima.

En resumen, hay muchos estudios sobre políticas empresariales medioambientales y sobre residuos sólidos, principalmente los que son generados por las actividades domésticas, sin embargo, no hay un análisis sobre si estas políticas de medio ambiente, incidan en la gestión de residuos en el sector construcción en el Perú, incluso internacionalmente hay poca información sobre este tema. Este es un primer análisis que prueba que una buena PEM debidamente implementada y soportada que incluya actividades de sostenibilidad y sustentabilidad, sensibilidad y educación ambiental de sus colaboradores, así como, responsabilidad social ambiental de las empresas constructoras, van a incidir positivamente en la GIRS. Este primer planteamiento se hace a través del conocimiento de los especialistas que fueron entrevistados y que nos permite augurar la reducción de los desperdicios de las obras a través del uso de buenos sistemas de gestión de proyectos, que incluya procesos de reciclamiento y en casos que no se pueda reciclar, que estos sean eliminados en forma correcta disponiéndolos en rellenos sanitarios autorizados. La consecuencia de mejorar la gestión de los residuos en construcción, es una menor afectación del medio ambiente mejorando la salud de la población en la ciudad de Lima.

\section{METODOLOGÍA}

El estudio constó de dos partes, la primera correspondió a un análisis estadístico para probar la correlación entre las dos variables (PEM y GIRS) y la segunda de un análisis de las respuestas de los entrevistados. El análisis estadístico tuvo un enfoque del tipo cuantitativo, para lo cual se elaboró una encuesta de 36 preguntas, las cuales se muestran en el anexo adjunto, dividida en dos secciones: la primera con 18 preguntas relacionadas con la variable independiente PEM, las cuales incluye temas sobre políticas de Sostenibilidad y Sustentabilidad Ambiental, Sensibilidad y Educación Ambiental y Responsabilidad Social Empresarial. La segunda también tiene 18 preguntas e incluye las interrogantes relacionadas con la variable dependiente GIRS, divididas en temas de Reducción, Tratamiento-Reciclamiento y Eliminación de los Residuos Sólidos. El detalle de la distribución de las preguntas en la encuesta se muestra en la tabla 2: Distribución de preguntas por variables.

La respuesta a cada pregunta de la encuesta está basada en la escala Likert cuya interpretación es el siguiente: 1= Nada, 2=Poco, 3=Regular, 4=Bastante y $5=$ Mucho.

También hay que indicar que la investigación es del tipo no experimental, TRANSVERSAL y CORRELACIONAL. Transversal debido que se realiza en un determinado momento buscando identificar lo

Tabla 2. Distribución de preguntas por variables

\begin{tabular}{|l|c|c|l|c|c|}
\hline \multicolumn{2}{|c|}{ Políticas empresariales medioambientales } & \multicolumn{2}{c|}{ Gestión integral de residuos sólidos } \\
\hline Dimensiones & Ítems & $\begin{array}{c}\text { Nro. } \\
\text { Preguntas }\end{array}$ & Dimensiones & $\begin{array}{c}\text { Nro. } \\
\text { Preguntas }\end{array}$ \\
\hline Sostenibilidad y Sustentabilidad & Del 1 al 6 & 6 & Reducción de residuos sólidos & Del 1 al 6 & 6 \\
\hline Sensibilidad y Educación Ambiental & Del 7 al 12 & 6 & $\begin{array}{l}\text { Tratamiento y reciclamiento de } \\
\text { residuos sólidos }\end{array}$ & Del 7 al 12 & 6 \\
\hline $\begin{array}{l}\text { Responsabilidad Social Ambiental } \\
\text { Empresarial (RSAE). }\end{array}$ & Del 13 al 18 & 6 & Eliminación de residuos sólidos & Del 13 al 18 & 6 \\
\hline Total, dimensión & $\mathbf{1 8}$ & Total, dimensión & 18 \\
\hline Total, encuesta
\end{tabular}

Fuente: Elaboración propia 
que está sucediendo con el problema planteado y Correlacional porque mide si las variables plateadas tienen relación entre sí, es decir, si la variable independiente, PEM tiene una correlación con la variable dependiente GIRS.

Esta encuesta fue aplicada a ejecutivos de empresas constructoras privadas con más de 100 trabajadores (medianas y grandes) dedicadas principalmente a la construcción de edificios, centros comerciales, viviendas, entre otras en la ciudad de Lima, ubicada en la costa central del Perú. La muestra fue de 86 profesionales de una población de 174 ejecutivos. La evaluación de esta encuesta para el primer análisis se realizó a través de métodos estadísticos utilizando el software de IBMSPSS versión 25.

Dentro de la rigurosidad de las pruebas estadísticas los resultados de la encuesta fueron sometida a los siguientes análisis: a) prueba de confiabilidad de la encuesta: Alpha Cronbach; b) prueba de normalidad de los resultados: Kolmogorov-Smirnov y c) prueba de contraste de hipótesis: Rho-Spearman.

La interpretación de los resultados de la prueba de hipótesis para ver si hay correlación entre las dos variables se dio de acuerdo con la escala mostrada en la tabla 3 Interpretación de la correlación de Hernández et al. (2010)

Siguiendo con el proceso de investigación y basándose en los problemas planteados definimos las variables independientes y dependientes, así como, sus dimensiones. i. Variable independiente: Políticas Empresariales Medioambientales (X),

Dimensiones: Sostenibilidad y Sustentabilidad Ambiental, sensibilidad y educación ambiental y responsabilidad social ambiental empresarial

ii. Variable dependiente: Gestión Integral de Residuos Sólidos (Y)

Dimensiones: Reducción de los Residuos Sólidos, tratamiento y Reciclamiento de los Residuos Sólidos y eliminación de los Residuos Sólidos

\section{RESULTADOS Y DISCUSIONES}

\section{Primera parte del estudio: Análisis estadístico}

En la prueba Alpha de Cronbach se obtuvo como resultado un coeficiente de fiabilidad, 0.914 para la variable PEM y 0.802 para la variable GIRS. Estos resultados son considerados como MUY ALTOS de acuerdo con la tabla de Pallella \& Martins, (2012, p. 169). De los resultados se deduce que el instrumento de medición usado, en este caso la encuesta, es consistente consigo mismo, es decir, tienen una buena correlación por lo que la encuesta es aceptada.

El siguiente paso es ver si los resultados obtenidos de la encuesta tienen una distribución normal. Es importante este análisis porque permite definir que test debe utilizarse en la prueba de hipótesis. Para ello aplicaremos la prueba de Kolmogorov-Smirnov para las dos variables:

Tabla 3. Interpretación de la correlación

\begin{tabular}{|c|l|c|l|}
\hline Correlación & \multicolumn{1}{|c|}{ Interpretación } & Correlación & \multicolumn{1}{|c|}{ Interpretación } \\
\hline-1.00 & $\begin{array}{l}\text { Correlación negativa perfecta ("A mayor } X, \text { menor } \\
\text { ", de manera proporcional. Es decir, cada vez } \\
\text { que } X \text { aumenta una unidad, } Y \text { disminuye siempre } \\
\text { una cantidad constante.) } \\
\text { Esto también se aplica "a menor } X, \text { mayor } Y \text { ”. }\end{array}$ & +0.10 & Correlación positiva muy débil \\
\hline-0.90 & Correlación negativa muy fuerte & +0.25 & Correlación positiva débil \\
\hline-0.75 & Correlación negativa considerable & +0.50 & Correlación positiva media \\
\hline-0.50 & Correlación negativa media & +0.75 & Correlación positiva considerable \\
\hline-0.25 & Correlación negativa débil & +0.90 & Correlación positiva muy fuerte \\
\hline-0.10 & Correlación negativa muy débil & +1.00 & $\begin{array}{l}\text { Correlación positiva perfecta ("A mayor } X, \text { mayor } Y \text { " } \\
\text { Cada vez que } X \text { aumenta, } Y \text { aumenta siempre una } \\
\text { cantidad constante.) }\end{array}$ \\
\hline 0.00 & No existe correlación alguna entre variables & & \\
\hline
\end{tabular}

Fuente: Hernández et al, 2010, Metodología de la Investigación, Quinta Edición, (p. 312) 
- Prueba de normalidad para la variable dependiente POLÍTICAS EMPRESARIALES MEDIOAMBIENTALES

Ho: La información obtenida de las Políticas Empresariales Medioambientales no es distinta a una distribución normal.

H1: La información obtenida de las Políticas Empresariales Medioambientales es distinta a una distribución normal.

Si $P<\alpha$, se rechaza $\mathrm{Ho}$, si $\mathrm{P}>\alpha$, no se rechaza $\mathrm{Ho}$, donde $\mathrm{P}$ es un valor de probabilidad que va entre 0 y 1 , este valor se compara con $\alpha$, el nivel de significancia, el cual para la prueba es de 0.05. Los resultados:

El resultado muestra una Sig. Asintótica (bilateral) igual a 0.000 , el cual es menor que 0.05 de la muestra, por lo tanto, se rechaza la hipótesis nula $(\mathrm{Ho})$ y se acepta la hipótesis alternativa (H1), "La información obtenida de las PEM es distinta a una distribución normal"

- Prueba de normalidad para la variable independiente GIRS

Ho: La información obtenida de la GIRS no es distinta a una distribución normal.

H1: La información obtenida de la GIRS es distinta a una distribución normal.

Si $P<\alpha$, se rechaza $\mathrm{Ho}$, si $P>\alpha$, no se rechaza $\mathrm{Ho}$, donde $\mathrm{P}$ es un valor de probabilidad que va entre 0 y 1 , este valor se compara con $\alpha$, el nivel de significancia, el cual para la prueba es de 0.05 .

El resultado para la Sig. Asintótica (bilateral) es igual a 0.000 , el cual es menor que 0.05 de la muestra, por lo tanto, al igual que la variable de Políticas, se rechaza la hipótesis nula $(\mathrm{Ho})$ y se acepta la hipótesis alternativa (H1), "La información obtenida de la GIRS es distinta a una distribución normal".

Del resultado de la prueba de normalidad para ambas variables se deduce que no es conocido el tipo de distribución (distribution free), por lo tanto, el estadístico aplicado para la prueba de hipótesis debería ser no paramétricas, siendo esta la "Rho de Spearman" la más adecuada.

\section{Prueba de HIPÓTESIS a contrastar:}

Nula $\left(\mathbf{H}_{0}\right)$ : Las Políticas Empresariales Medioambientales no inciden en la GIRS en las obras del sector construcción en Lima Metropolitana.

Alternativa $\left(\mathbf{H}_{1}\right)$ : Las Políticas Empresariales Medioambientales si inciden en la GIRS en las obras del sector construcción en Lima Metropolitana.

Los resultados obtenidos después de utilizar el software IBM-SPSS se muestran en la tabla 4 Prueba de hipótesis de las variables generales, mostrada líneas abajo.

El resultado obtenido muestra una "correlación positiva considerable" de 0.668 entre las dos variables según la interpretación de Hernández et al. (2010) mostrado en la tabla 3 . Por otro lado, el nivel de Sig. (bilateral) es 0.000 es menor al nivel de significancia de la prueba 0.05 , por lo tanto, se rechaza la Hipótesis Nula $\left(\mathrm{H}_{0}\right)$; como consecuencia se acepta la hipótesis alternativa que indica las PEM si inciden en la GIRS.

\section{Segunda parte del estudio: Análisis de las respuestas}

Si bien el resultado obtenido muestra una correlación positiva considerable, el valor no es muy alto,

Tabla 4. Prueba de la hipótesis de las variables generales

\begin{tabular}{|l|l|l|c|c|}
\hline \multicolumn{2}{|c|}{ Correlaciones } & \multicolumn{1}{c|}{ PromPoliticas } & PromResiduos \\
\hline \multirow{3}{*}{ Rho de Spearman } & \multirow{2}{|c|}{ PromPoliticas } & Coeficiente de correlación & 1.000 & $.668^{* *}$ \\
\cline { 3 - 5 } & & Sig. (bilateral) &. & .000 \\
\cline { 3 - 5 } & & $\mathrm{N}$ & .66 & 86 \\
\cline { 2 - 5 } & \multirow{2}{*}{ PromResiduos } & Coeficiente de correlación & .000 & 1.000 \\
\cline { 3 - 5 } & & Sig. (bilateral) & 86 & 86 \\
\cline { 3 - 5 } & & $\mathrm{N}$ & &. \\
\hline
\end{tabular}

**. La correlación es significativa en el nivel 0.01 (bilateral).

Fuente: Resultado software de IBM-SPSS versión 25 
por lo que se vuelve importante realizar un segundo análisis revisando los resultados del estudio y ver cuál es la frecuencia de las respuestas, según la escala Likert indicada anteriormente. De las respuestas se pueden deducir varias conclusiones, en este artículo revisaremos solo la más votadas para cada variable, es decir, determinar qué respuesta tienen mayor fuerza, en opinión de los entrevistados y así ampliar las conclusiones y las posibles recomendaciones, en apoyo a una buena gestión de los residuos sólidos en los proyectos del sector construcción en la ciudad de Lima Metropolitana.
Basándose en lo indicado, las frecuencias obtenidas en las dos variables son los siguientes:

Analizando la tabla 5 Frecuencia de las variables, podemos determinar cuáles son las preguntas que han sido votados con mayor frecuencia y a la escala Likert que pertenece. Los resultados son los siguientes:

En las preguntas de la variable PEM en la dimensión Sensibilidad y Educación Ambiental tenemos: con $62 \%$ en la escala "Bastante" la pregunta 11

Tabla 5. Frecuencias de las variables

\begin{tabular}{|c|c|c|c|c|c|c|c|c|c|c|c|c|c|c|c|c|c|c|}
\hline \multicolumn{19}{|c|}{ Frecuencia de la variable Políticas Empresariales Medioambientales } \\
\hline \multirow{2}{*}{ \# pregunta } & \multicolumn{6}{|c|}{ Sostenibilidad y Sustentabilidad } & \multicolumn{6}{|c|}{ Sensibilidad y Educación ambiental } & \multicolumn{6}{|c|}{ Responsabilidad Social } \\
\hline & 1 & 2 & 3 & 4 & 5 & 6 & 7 & 8 & 9 & 10 & 11 & 12 & 13 & 14 & 15 & 16 & 17 & 18 \\
\hline Fre & $\mathrm{F}$ & $\mathrm{F}$ & $\mathrm{F}$ & $r$ & $r$ & $r$ & $\mathrm{~F}$ & $\mathrm{~F}$ & $\mathrm{~F}$ & $\mathrm{~F}$ & $\mathrm{~F}$ & $\mathrm{~F}$ & $\mathrm{~F}$ & $r$ & $\mathrm{~F}$ & $\mathrm{~F}$ & $\mathrm{~F}$ & $\mathrm{~F}$ \\
\hline \multirow{2}{*}{ Nada } & 3 & 3 & 2 & 1 & 6 & 1 & 2 & 8 & 6 & 7 & 4 & 2 & 2 & 0 & 6 & 4 & 0 & 0 \\
\hline & $3 \%$ & $3 \%$ & $2 \%$ & $1 \%$ & $7 \%$ & $1 \%$ & $2 \%$ & $9 \%$ & $7 \%$ & $8 \%$ & $5 \%$ & $2 \%$ & $2 \%$ & $0 \%$ & $7 \%$ & $5 \%$ & $0 \%$ & $0 \%$ \\
\hline \multirow{2}{*}{ Poco } & 3 & 5 & 21 & 3 & 22 & 6 & 10 & 23 & 12 & 7 & 3 & 0 & 7 & 2 & 39 & 36 & 1 & 1 \\
\hline & $3 \%$ & $6 \%$ & $24 \%$ & $3 \%$ & $26 \%$ & $7 \%$ & $12 \%$ & $27 \%$ & $14 \%$ & $8 \%$ & $3 \%$ & $0 \%$ & $8 \%$ & $2 \%$ & $45 \%$ & $42 \%$ & $1 \%$ & $1 \%$ \\
\hline \multirow{2}{*}{ Regular } & 8 & 28 & 35 & 22 & 28 & 27 & 28 & 11 & 18 & 23 & 7 & 18 & 15 & 10 & 24 & 43 & 8 & 12 \\
\hline & $9 \%$ & $33 \%$ & $41 \%$ & $26 \%$ & $33 \%$ & $31 \%$ & $33 \%$ & $13 \%$ & $21 \%$ & $27 \%$ & $8 \%$ & $21 \%$ & $17 \%$ & $12 \%$ & $28 \%$ & $50 \%$ & $9 \%$ & $14 \%$ \\
\hline \multirow{2}{*}{ Bastante } & 51 & 32 & 18 & 47 & 25 & 42 & 33 & 40 & 44 & 41 & 53 & 52 & 48 & 52 & 17 & 3 & 50 & 48 \\
\hline & $59 \%$ & $37 \%$ & $21 \%$ & $55 \%$ & $29 \%$ & $49 \%$ & $38 \%$ & $47 \%$ & $51 \%$ & $48 \%$ & $62 \%$ & $60 \%$ & $56 \%$ & $60 \%$ & $20 \%$ & $3 \%$ & $58 \%$ & $56 \%$ \\
\hline \multirow{2}{*}{ Mucho } & 21 & 18 & 10 & 13 & 5 & 10 & 13 & 4 & 6 & 8 & 19 & 14 & 14 & 22 & 0 & 0 & 27 & 25 \\
\hline & $24 \%$ & $21 \%$ & $12 \%$ & $15 \%$ & $6 \%$ & $12 \%$ & $15 \%$ & $5 \%$ & $7 \%$ & $9 \%$ & $22 \%$ & $16 \%$ & $16 \%$ & $26 \%$ & $0 \%$ & $0 \%$ & $31 \%$ & $29 \%$ \\
\hline \multirow{2}{*}{ Total } & 86 & 86 & 86 & 86 & 86 & 86 & 86 & 86 & 86 & 86 & 86 & 86 & 86 & 86 & 86 & 86 & 86 & 86 \\
\hline & $100 \%$ & $100 \%$ & $100 \%$ & $100 \%$ & $100 \%$ & $100 \%$ & $100 \%$ & $100 \%$ & $100 \%$ & $100 \%$ & $100 \%$ & $100 \%$ & $100 \%$ & $100 \%$ & $100 \%$ & $100 \%$ & $100 \%$ & $100 \%$ \\
\hline \multicolumn{19}{|c|}{ Frecuencia de la variable Gestión Integral de los Residuos Sólidos } \\
\hline \multirow{2}{*}{ \# pregunta } & \multicolumn{6}{|c|}{ Reducción de los RS } & \multicolumn{6}{|c|}{ Tratamiento y reciclamiento RS } & \multicolumn{6}{|c|}{ Eliminación de RS } \\
\hline & 1 & 2 & 3 & 4 & 5 & 6 & 7 & 8 & 9 & 10 & 11 & 12 & 13 & 14 & 15 & 16 & 17 & 18 \\
\hline cia & $\mathrm{F}$ & $\mathrm{F}$ & $\mathrm{F}$ & $\mathrm{F}$ & $\mathrm{F}$ & $\mathrm{F}$ & $\mathrm{F}$ & $\mathrm{F}$ & $\mathrm{F}$ & $\mathrm{F}$ & $\mathrm{F}$ & $\mathrm{F}$ & $\mathrm{F}$ & $\mathrm{F}$ & $F$ & $F$ & $F$ & $\mathbf{F}$ \\
\hline \multirow{2}{*}{ Nada } & 0 & 0 & 0 & 0 & 0 & 0 & 0 & 3 & 3 & 0 & 4 & 0 & 7 & 9 & 1 & 0 & 53 & 12 \\
\hline & $0 \%$ & $0 \%$ & $0 \%$ & $0 \%$ & $0 \%$ & $0 \%$ & $0 \%$ & $3 \%$ & $3 \%$ & $0 \%$ & $5 \%$ & $0 \%$ & $8 \%$ & $10 \%$ & $8 \%$ & $9 \%$ & $62 \%$ & $14 \%$ \\
\hline \multirow{2}{*}{ Poco } & 1 & 4 & 1 & 0 & 2 & 2 & 0 & 17 & 14 & 3 & 9 & 2 & 8 & 21 & 47 & 5 & 0 & 31 \\
\hline & $1 \%$ & $5 \%$ & $1 \%$ & $0 \%$ & $2 \%$ & $2 \%$ & $0 \%$ & $20 \%$ & $16 \%$ & $3 \%$ & $10 \%$ & $2 \%$ & $9 \%$ & $24 \%$ & $55 \%$ & $6 \%$ & $0 \%$ & $36 \%$ \\
\hline \multirow{2}{*}{ Regular } & 5 & 11 & 17 & 16 & 5 & 19 & 25 & 8 & 11 & 24 & 5 & 3 & 7 & 12 & 10 & 5 & 7 & 18 \\
\hline & $6 \%$ & $13 \%$ & $20 \%$ & $19 \%$ & $6 \%$ & $22 \%$ & $29 \%$ & $9 \%$ & $13 \%$ & $28 \%$ & $6 \%$ & $3 \%$ & $8 \%$ & $14 \%$ & $12 \%$ & $6 \%$ & $8 \%$ & $21 \%$ \\
\hline \multirow{2}{*}{ Bastante } & 50 & 49 & 45 & 55 & 63 & 54 & 58 & 58 & 56 & 48 & 46 & 55 & 43 & 23 & 22 & 38 & 26 & 23 \\
\hline & $58 \%$ & $57 \%$ & $52 \%$ & $64 \%$ & $73 \%$ & $63 \%$ & $67 \%$ & $67 \%$ & $65 \%$ & $56 \%$ & $53 \%$ & $64 \%$ & $50 \%$ & $27 \%$ & $26 \%$ & $44 \%$ & $30 \%$ & $27 \%$ \\
\hline \multirow{2}{*}{ Mucho } & 30 & 22 & 23 & 15 & 16 & 11 & 3 & 0 & 2 & 11 & 22 & 26 & 21 & 21 & 0 & 30 & 0 & 2 \\
\hline & $35 \%$ & $26 \%$ & $27 \%$ & $17 \%$ & $19 \%$ & $13 \%$ & $3 \%$ & $0 \%$ & $2 \%$ & $13 \%$ & $26 \%$ & $30 \%$ & $24 \%$ & $24 \%$ & $0 \%$ & $35 \%$ & $0 \%$ & $2 \%$ \\
\hline \multirow{2}{*}{ Total } & 86 & 86 & 86 & 86 & 86 & 86 & 86 & 86 & 86 & 86 & 86 & 86 & 86 & 86 & 86 & 86 & 86 & 86 \\
\hline & $100 \%$ & $100 \%$ & $100 \%$ & $100 \%$ & $100 \%$ & $100 \%$ & $100 \%$ & $100 \%$ & $100 \%$ & $100 \%$ & $100 \%$ & $100 \%$ & $100 \%$ & $100 \%$ & $100 \%$ & $100 \%$ & $100 \%$ & $100 \%$ \\
\hline
\end{tabular}

Nota: F=Frecuencia, RS=Residuo sólido

Fuente: Elaboración propia basándose en los resultados de la encuesta 
¿Cree que utilizando las últimas herramientas de gestión ayudará mejorar la gestión del medio ambiente?

Dentro de las recomendaciones dadas por los entrevistados está el uso de la filosofía Lean Construcción, en sus actividades, también están los conceptos del Project Management Body of Knowledge (PMBOK) del PMI o utilizar el Building Information Modeling (BIM)

En las preguntas de GIRS en la dimensión Reducción de los Residuos Sólidos tenemos: con $73 \%$ en la escala Bastante la pregunta 5 ¿Cree Ud. que se puede reducir los desperdicios sólidos en la etapa de Diseño del proyecto? Los entrevistados en su mayoría están de acuerdo que un buen diseño del proyecto hace eficiente el proceso constructivo y ayuda a reducir los residuos sólidos. Al respecto Capó (2005) menciona la necesidad de la participación del contratista en la etapa de diseño lo cual fue planteado también por los encuestados: "Si los contratistas no se involucran en la pre-construcción, el equipo de diseño puede encontrarse con el problema de tener que rediseñar alguna solución constructica, una vez que el proyecto este en marcha" (p.24)

\section{CONCLUSIONES Y RECOMENDACIONES}

De los resultados de la encuesta se puede concluir lo siguiente:

1. Se ha confirmado el objetivo general, es decir, hay incidencia positiva entre las PEM y la GIRS en las obras del sector construcción en Lima Metropolitana. Esto es comprobado a través de los resultados de la prueba estadística Rho de Spearman aplicada sobre las variables objetivos del estudio, dando como resultado un puntaje de 0.668 , siendo esta una "correlación positiva significativa" según la escala de Hernández et al. (2010, p.312).

La interpretación de estos resultados está dada por la necesidad de que las PEM de las empresas constructoras no sean simplemente una declaración de buenas intenciones puesta en un anuario o en la página web de la organización, sino que tomen un rol importante en el desarrollo de sus actividades, gestionando correctamente los residuos sólidos de las obras. El beneficio para la sociedad está dado en la descontaminación del medio ambiente de Lima a través de la la reducción de los residuos mediante la reutilizados, reciclamiento o correcta eliminación de los mismos, según las pautas comentadas en el presente artículo.
2. Las mejoras que se obtengan con el manejo de los residuos deben ser sostenibles y sustentables en el tiempo, lo cual se puede obtener a través de sensibilización de sus colaboradores en la protección del medio ambiente, educándolos y haciendo que las empresas asuman sus compromisos ambientales como indica el PNUMA de la ONU

3. También hay conclusiones que son tomadas de las conversaciones con los entrevistados, los cuales no se pueden plasmar en un número estadístico. Una de ellas es, como hacer que las políticas empresariales, en este caso medioambientales, sean efectivas o positivas en el manejo de los residuos sólidos en las obras. Aquí es importante analizar el código de ética de la organización y el modo de pensar de la alta gerencia, si esta no es sensible con su medio ambiente, es poco lo que pueda avanzar en este camino. Hay que tener en cuenta que la ética de las organizaciones está muy relacionada con su responsabilidad social ambiental reflejada en la misión y visión de la empresa. Aunque parece una pregunta evidente, se ha demostrado que la responsabilidad social tiene una significancia importante en los ejecutivos entrevistados.

4. El uso de modernas herramientas de gestión en la ejecución de los proyectos ayudaría a ser más eficientes en el manejo de los residuos de las obras de construcción. Es opinión de los entrevistados que los proyectos en Lima se hacen con poco planeamiento generando reprocesos y cambios de especificación técnicas constantemente, lo cual produce un exceso de residuos, que luego son mal eliminados. Se propone dar énfasis en la implementación del sistema de planeamiento (último planificador) de la filosofía del Lean Construcción donde su principal principio es la eliminación de pérdidas en los proyectos, es decir, eliminar de lo que no agrega valor al producto. Otra herramienta mencionada es la aplicación de los conceptos del Project Management Institute (PMI) a través del Project Management Body of Knowledge (PMBOK) y la certificación para los profesionales dedicados a la ejecución de proyectos, entre ellos, la construcción de obras. Este debe ser un requisito obligatorio para todos los profesionales que lideren la ejecución un proyecto. Otra herramienta es el uso del Building Information Modeling (BIM) que ya está siendo obligatorio en algunas empresas como método de trabajo aplicados al diseño y la gestión de 
los proyectos. La utilización de estas nuevas herramientas mejora la gestión de los residuos a través de un buen diseño de los proyectos, correcta especificación de los materiales y una buena logística tanto interna como externa a la obra. El uso de estas herramientas y otras más apoya la eficiencia de la gestión de los residuos sólidos y como consecuencia una menor afectación del medio ambiente en la ciudad de Lima.

5. Con respecto a la variable GIRS, la pregunta con mayor relevancia, según los encuestados fue la 5 (73\%, bastante) ¿Cree Ud. que se puede reducir los desperdicios sólidos en la etapa de Diseño del proyecto? Los entrevistados en su mayoría están de acuerdo que un buen diseño del proyecto hace más eficiente el proceso constructivo y plantearon la necesidad de que participe el contratista en esta etapa por sus conocimientos de la realidad en el suministro de materiales y servicios, entre otros elementos, que ayudan a una mejor ejecución de la obra produciendo menos errores y a la vez menos desperdicios.

\section{REFERENCIAS BIBLIOGRÁFICAS}

[1] AMBIDES, (2012). Informe Anual de Residuos Sólidos Municipales y no Municipales en el Perú, Gestión 2012. Lima.

[2] Capó J. (2005). Gestión del Conocimiento de la Cadena de Suministro de la Construcción. Directivos Construcción, 26.

[3] Observatorio ciudadano, Lima cómo Vamos, (2015). Evaluando la Gestión en Lima: Quinto Informe de Resultado sobre Calidad de Vida. Lima: Asociación UNACEM.

[4] Dourojeanni, A. (1999). Dinámica del desarrollo sustentable y sostenible. CEPAL - Comisión Económica para América Latina y el Caribe, 1-26.

[5] Durand, M., y Metzger, P. (2009). Gestión de residuos y transferencia de vulnerabilidad en Lima/Callao. I'Institut Francais d'Etudes Andines. Lima: IFEA.
[6] Hernández S R, Fernandez CC., Baptista L M. del P. (2010). Metodología de la Investigación. México D.F.: McGraw Hill - Interamericana Editores SA de CV.

[7] INEI, I. N. (2018). Perú: Anuario de Estadísticas Ambientales 2018. Lima: INEI.

[8] Koontz y O'Donell, (1972). Curso de Administración Moderna: un análisis de las funciones de la administración. México: McGraw-Hill.

[9] Larrouyet C, (2015). Desarrollo sustentable: origen, evolución y su implementación para el cuidado del planeta. Tesis posgrado, Universidad Nacional de Quilmes, Buenos Aires.

[10] Ministerio del Ambiente. (28 de Febrero de 2017). D.L. 1278 Ley de Gestión Integral de Residuos Sólidos. Lima, Lima, Perú: Diario el Peruano.

[11] Ministerio del Ambiente. (21 de Diciembre de 2017). DS 014-2017-MINAN. Reglamento de la Ley de Gestión Integral de Residuos Solidos. Lima, Lima, Perú: Diario el Peruano.

[12] Pallella S., \& Martíns F (2012), Metodología de la Investigación Cuantitativa (tercera ed.). Caracas, Venezuela: Editorial pedagógica de Venezuela.

[13] Pulgar M, (2010). Responsabilidad social y política ambiental: complementariedades y retos. Revista de la Facultad de Derecho PUCP, 217 - 229.

[14] Rivas M, (2011). Modelo de sistema de gestión ambiental para formar universidades ambientalmente sostenibles en Colombia. Gestión y Ambiente, 151-161.

[15] Rodríguez N, (11 de Noviembre de 2018). Introducing Environmental Corporation Social Responsability. ClimBlog. 


\section{ANEXO}

\section{Preguntas de la encuesta}

A. Políticas Empresariales Medioambientales - PEM (X)

Sostenibilidad y Sustentabilidad Ambiental $\left(X_{1}\right)$

1. ¿Cuánto conoce usted las PEM de su organización?

2. ¿Está de acuerdo que las PEM garantizan la integridad del medio ambiente a largo plazo?

3. ¿Qué tan difícil es aplicar las PEM en sus proyectos?

4. ¿Cree que las constructoras deben preocuparse en conservar el medio ambiente en la ciudad de Lima?

5. ¿Los estudios de impacto ambiental de los proyectos, mejora el medio ambiente en la ciudad de Lima?

6. ¿En las PEM de su organización, se debe promover el uso de energía renovable en sus proyectos?

Sensibilidad y Educación Ambiental $\left(\mathbf{X}_{2}\right)$

7. ¿Cree Ud. que proteger el medio ambiente es necesario, aunque ello implique un menor desarrollo económico para la organización?

8. ¿Las PEM de su organización apoyan en sensibilizar a sus colaboradores para no deteriorar el medio ambiente que lo rodea?

9. ¿En qué medida las PEM de su organización sensibiliza a sus subcontratistas para no afectar su medio ambiente?

10. ¿Las charlas de 5 minutos sobre mejorar el medio ambiente, tendría efecto positivo en sus colaboradores?

11. ¿Cree que utilizando las últimas herramientas de gestión ayudará mejorar la gestión del medio ambiente?

12. ¿Cree que sus stakeholders de sus proyectos afectarían la aplicación de sus políticas de medio ambientales?

Responsabilidad Social Ambiental Empresarial (X3)

13. ¿Cuánto conoce usted sobre RSA, como protección del medio ambiente?

14. ¿Está de acuerdo que las normas de RSA deben estar relacionadas con Misión y Visión de la organización?

15. ¿Conoce Ud. la postura sobre la RSA de sus proveedores, socios estratégicos y subcontratistas?

16. ¿En qué medida su organización, a través de sus PEM, difunden la RSA en la sociedad?

17. ¿Cree usted que existe en su organización conciencia colectiva sobre la protección del medio ambiente?

18. ¿En su opinión, el promover la RSA afecta positivamente la competitividad de su organización en el mercado?

\section{B. Gestión Integral de Residuos Sólidos - GIRS (Y)}

Reducción de los Residuos Sólidos (Y1)

1. ¿En qué medida, las improvisaciones en un proyecto contribuyen a aumentar los residuos en construcción?

2. ¿Cuánto conocimiento tiene sobre las distintas formas de reducir los residuos sólidos en construcción?

3. ¿Mejoraría tener dentro de la organización un área que gestione los residuos de sus proyectos?

4. ¿Qué conocimiento tiene de herramientas de gestión que apoyen en reducir los residuos sólidos en sus obras?

5. ¿Cree Ud. que se puede reducir los desperdicios sólidos en la etapa de Diseño del proyecto?

6. ¿Capacitando a sus colaboradores se podrá reducir los residuos sólidos en sus proyectos?

Tratamiento y Reciclamiento de los Res. Sólidos $\left(\mathrm{Y}_{2}\right)$

7. ¿Qué tanto conocimiento tiene Ud. que los residuos sólidos de las obras se pueden reciclar?

8. ¿Con que frecuencia recicla materiales residuales en sus proyectos?

9. ¿Usa contenedores para reciclar materiales en sus proyectos?

10. ¿Qué tanto conocimiento tiene que el concreto y tabiquería se puede reciclar?

11. ¿Utiliza Ud. productos reciclados en sus proyectos?

12. ¿Sabe Ud. que se puede obtener un beneficio económico para el proyecto, reciclando sus residuos sólidos?

Eliminación de los Residuos Sólidos $\left(\mathrm{Y}_{3}\right)$

13. ¿Participa en la eliminación de los residuos sólidos de sus proyectos?

14. ¿En qué medida conoce Ud. las zonas de botaderos de la ciudad de Lima?

15. ¿Conoce Ud. empresas que tengan "Plan de Manejo de Residuos Sólidos no Peligros"?

16. ¿Qué tanto estaría dispuesto a ubicar áreas dentro de su proyecto para acumular los residuos sólidos que van a ser eliminados?

17. ¿Conoce Ud. empresas constructoras que eliminen sus residuos sólidos en botaderos no autorizados?

18. ¿Conoce usted la reglamentación del estado sobre la eliminación de residuos sólidos en Lima Metropolitana? 